\title{
O impacto da endometriose na saúde física e mental da mulher
}

\author{
The impact of endometriosis on women's physical and mental health
}

El impacto de la endometriosis en la salud física y mental de las mujeres

Camila Caires Brito ${ }^{1 *}$, Murillo Cursino de Castro Silva ${ }^{2}$, Patricia Lelis Marques ${ }^{2}$, Rodrigo Freitas Parrela $^{3}$, Evelyn Silva Souza ${ }^{3}$, Bruna de Aquino Morais da Silva ${ }^{2}$, Louise Leandro Carneiro ${ }^{3}$, Camila Freitas Barbosa ${ }^{2}$, Victor Uelcio Cangussu de Assis ${ }^{2}$, Eugênia Ferraz Silva ${ }^{1}$.

\section{RESUMO}

Objetivo: Entender como a endometriose impacta na saúde física e mental da mulher. Revisão bibliográfica: A endometriose é definida como a presença de glândulas e estroma endometriais fora do sítio normal. As manifestações clínicas se apresentam de forma heterogênea, que podem ser assintomáticas até extremamente incapacitantes. A doença atinge cerca de $15 \%$ das mulheres no menacme e $70 \%$ destas apresentam dor pélvica crônica; Sendo as dores frequentes a principal queixa, podendo levar a perdas de dias de trabalho e isso pode influenciar negativamente na sua carreira e financeiramente, trazendo prejuízos gerais na vida da paciente. Uma vez que, a maioria das mulheres nunca ouviu falar da doença antes de chegar ao diagnóstico, e desde o início já começa a experimentar uma série de frustrações e conflitos como raiva, angústia, ansiedade e medo. Considerações finais: A endometriose atualmente é uma doença muito prevalente entre as mulheres, e que ainda representa um desafio para os ginecologistas por ser uma doença pouco conhecida e na maioria das vezes apresentar atraso no diagnóstico, sendo essa falta de informação uma das principais queixas das portadoras desta patologia.

Palavras-chave: Endometriose, Saúde física, Saúde mental.

\begin{abstract}
Objective: To understand how endometriosis impacts women's physical and mental health. Review bibliographic: Endometriosis is defined as the presence of endometrial glands and stroma outside the normal site. Clinical manifestations are heterogeneous, which can be asymptomatic to extremely disabling. The disease affects about $15 \%$ of women at menacme and $70 \%$ of these have chronic pelvic pain. Being frequent pain the main complaint, which can lead to loss of work days and this can negatively influence your career and financially, bringing general damage to the patient's life. Since most women have never heard of the disease before reaching the diagnosis, and from the beginning they already start to experience a series of frustrations and conflicts such as anger, anguish, anxiety and fear. Final considerations: Endometriosis is currently a disease It is very prevalent among women, and it still represents a challenge for gynecologists because it is a little known disease and most of the times it presents a delay in diagnosis. This lack of information is one of the main complaints of patients with this pathology.
\end{abstract}

Key words: Endometriosis, Physical health, Mental health.

\section{RESUMEN}

Objetivo: Comprender cómo la endometriosis afecta la salud física y mental de las mujeres. Revisión bibliográfica: La endometriosis se define como la presencia de glándulas endometriales y estroma fuera del

\footnotetext{
${ }^{1}$ Faculdade Santo Agostinho (FASA), Vitória da Conquista - BA. *E-mail: camila_crvg@hotmail.com

2 Centro Universitário FG (UniFG), Guanambi - BA.

${ }^{3}$ Faculdade Santo Agostinho de Itabuna (FASAI), Itabuna - BA.
} 
sitio normal. Las manifestaciones clínicas son heterogéneas, que pueden ser asintomáticas 0 extremadamente discapacitantes. La enfermedad afecta aproximadamente al 15\% de las mujeres en menacme y el $70 \%$ de ellas tienen dolor pélvico crónico. Siendo el dolor frecuente la principal queja, que puede derivar en la pérdida de jornadas laborales y esto puede influir negativamente en su carrera y económicamente, trayendo daños generales a la vida del paciente. Dado que la mayoría de las mujeres nunca habían oído hablar de la enfermedad antes de llegar al diagnóstico, y desde el principio ya comienzan a experimentar una serie de frustraciones y conflictos como la ira, la angustia, la ansiedad y el miedo. Consideraciones finales: La endometriosis es actualmente una enfermedad Es muy prevalente entre las mujeres, y aún representa un desafío para los ginecólogos porque es una enfermedad poco conocida y la mayoría de las veces presenta un retraso en el diagnóstico, y esta falta de información es una de las principales quejas de los pacientes con esta patología.

Palabras clave: Endometriosis, Salud física, Salud mental.

\section{INTRODUÇÃO}

A Endometriose (EDM) é uma ginecopatia de caráter progressivo que ocorre em mulheres, principalmente naquelas em idade reprodutiva e pode ser caracterizada quando há presença de endométrio em locais ectópicos. O tecido endometrial pode acometer várias regiões fora da cavidade uterina, como, por exemplo, ovários, peritônio, ligamentos úterossacros, região retro-cervical, septo reto-vaginal, reto/sigmoide, íleo terminal, apêndice, bexiga e ureteres (ARAÚJO FWC e SCHMIDT DB, 2020).

Suas consequências são variáveis de acordo com sua localização, que podem ser: dismenorreia (cólica menstrual), algia pélvica crônica (dor), infertilidade, dispareunia (dor genital durante ou após o ato sexual), alterações intestinais e urinárias cíclicas, como dor à evacuação, diarreia, disúria perimenstrual (dor durante a micção no período menstrual), polaciúria (aumento da frequência miccional), urgência miccional e hematúria (emissão de sangue através da uretra, acompanhado ou não pela urina). Por ser considerada uma doença crônica, os sintomas persistentes podem causar prejuízos em diversas esferas da vida da mulher, tais como, físico, psíquico e social, contribuindo para a redução da sua qualidade de vida. Essa redução pode ser explicada pela complexidade da etiologia e manifestação dessa doença (TOMÁs C e METELLO JL, 2019).

As manifestações clínicas da EDM afetam a vida das pacientes, sendo as dores frequentes a principal queixa que gera limitações, podendo levar a perdas de dias de trabalho e isso pode influenciar negativamente na sua carreira e financeiramente, trazendo prejuízos gerais na vida da paciente. Isso ocorre principalmente devido a uma redução da capacidade para exercer sua função, por absenteísmo devido à dor e ou internamento hospitalar ou por falta de capacidades cognitivas e psicológicas para desenvolver as suas tarefas laborais (MORAIS RL e ROSAL MA, 2021).

Além disso, a EDM é uma das principais causas da infertilidade feminina; Causa esta que afeta laços familiares e conjugal, uma vez que essas mulheres não se sentem confiantes de que vão ser compreendidas quanto aos seus sintomas e porque este é um assunto difícil. A doença também restringe e modifica o convívio diário da mulher com suas rotinas, limitando a sua qualidade de vida, o que reflete, principalmente, na sua autoestima. Nesse sentido, a diminuição percebida da satisfação sexual e do bem-estar pessoal pode justificar o fato dessas mulheres atribuírem à sua doença uma razão para o divórcio civil e ao distanciamento social (OLIVEIRA LAF, et al., 2018).

A EDM pode ainda cursar com alterações emocionais de dúvidas e incertezas, uma vez que, a maioria das mulheres nunca ouviu falar da doença antes de chegar ao diagnóstico, e desde o início já começa a experimentar uma série de frustrações e conflitos como raiva, angústia, ansiedade, medo. Por isso, a maioria das mulheres com endometriose tem dificuldade em planejar o futuro, pois vive com receio da dor, da infertilidade e com receio de falta de apoio social por ser considerada "menos mulher", por não poder ter filhos ou por estar com dores constantes (ARAÚJO FWC e SCHMIDT DB, 2020).

O curso da doença tem caráter ainda enigmático, devido a sua etiopatogenia incerta e tratamento variável, Isto reforça o paralelo entre a relação do perfil psicológico e a intensidade da dor relatada pelas pacientes, sugerindo a existência de uma desordem na personalidade. Diante disso, encontramos a depressão e a 
ansiedade associados à EDM, e se não diagnosticadas, interferem no tratamento, levando a uma piora da doença. Essas alterações de humor nas mulheres portadoras dessa patologia também estão relacionadas à busca incessante pelo tratamento das dores incapacitantes, à demora do diagnóstico e à incapacidade no manejo da dor (BAETAS BV, et al., 2021).

Associadas a esse quadro de depressão e ansiedade, algumas manifestações são comuns nessas mulheres como, por exemplo, humor deprimido, diminuição acentuada do interesse e do prazer pelas atividades anteriormente satisfatórias, perda ou aumento significativo de peso, insônia ou hipersonia, agitação ou retardo psicomotor, fadiga, pensamentos de morte, entre outros. Isso impacta negativamente na maneira da paciente lidar com a dor, no seu quotidiano e principalmente na sua qualidade de vida, fazendo com que a paciente se torne isolada, prejudicando os seus relacionamentos (YELA DA, et al., 2020).

Alguns sintomas clínicos da EDM podem levar anos para que a evolução se torne aparente, com o diagnóstico questionado por ser muitas vezes realizado tardiamente. Por isso, o cuidado à mulher com endometriose não se resume apenas no manejo dos sintomas da patologia orgânica, mas significa também avaliá-la integralmente, o que inclui o seu estado emocional. Sendo assim, visto que o curso natural da doença pode ser alterado pelos sintomas psíquicos, é de extrema importância relaciona-los com o desenvolvimento dessa patologia, buscando um tratamento multiprofissional dos sintomas no intuito de melhorar sua condição física, psíquica e social (SOUZA TSB, et al., 2019).

Assim, esse trabalho teve como objetivo entender como a endometriose impacta na saúde física e mental da mulher.

\section{REVISÃO BIBLIOGRÁFICA}

A endometriose é um distúrbio ginecológico benigno definido como a presença de glândulas e estroma endometriais fora do sítio normal uterino. Como esse tecido endometrial é igual histologicamente ao endométrio, o crescimento e a manutenção desses implantes endometriais são dependentes da ação hormonal. Sendo assim, é mais comum o surgimento da EDM nas mulheres em idade reprodutiva, sendo rara em pré-púberes e no climatério (MORAIS RL e ROSAL MA, 2021).

A doença atinge cerca de $15 \%$ das mulheres no menacme e $70 \%$ destas apresentam dor pélvica crônica, sendo essa caracterizada ainda, em dismenorreia, dispareunia profunda e Dor Pélvica Crónica não menstrual (DPC). Além disso, até 50\% das mulheres com infertilidade possuem endometriose, e $50 \%$ com endometriose são inférteis. Essa patologia é considerada atualmente como a doença da mulher moderna, uma vez que cursa com padrões comuns à vida atual da mulher, principalmente 0 adiamento da primeira gestação (NOGUEIRA ACR, et al., 2018).

As manifestações clínicas se apresentam de forma heterogênea, de assintomáticas até extremamente incapacitantes. Correspondem a fenótipos distintos, porém com características histológicas comuns: presença de células de origem endometrial fora da cavidade uterina, com sinais de inflamação e sangramento de caráter crônico. Sendo assim, existe uma correlação entre o quadro clínico dessas pacientes com a localização das lesões endometriais, sua intensidade e profundidade (SOUZA GKT, et al., 2017).

A maior dificuldade dessas mulheres é conviver com a dor, sendo esta manifestação a mais observada; Sentem-se desvalorizadas quanto às suas queixas, e à percepção de ter seus sintomas ignorados, principalmente por parte de quem esperam ter apoio. Além da própria doença agravar a saúde física da mulher, por limitações impostas principalmente pela dor, essas mulheres se sentem também impotentes tanto no âmbito laboral, quanto social e afetivo, prejudicando sua saúde mental (SILVA CM, et al., 2021).

Sendo assim, a capacidade de trabalho, relações familiares e interpessoais, autoestima e humor são afetadas na vida da mulher com EDM, diminuindo sua qualidade de vida, além de ser grande causa de incapacidade. A influência negativa que esses aspectos causam no bem-estar dessas pacientes acarreta 0 isolamento social. Essa diminuição da qualidade de vida está relacionada com a complexidade da fisiopatologia da doença e como ela se manifesta, além da dúvida sobre a responsividade das intervenções e suas consequências, como a interferência direta na capacidade reprodutiva da mulher atrelada a dor crônica (MORAIS HB, et al., 2019). 
Os sintomas incluem um caráter crônico, com aderências e inflamações que podem aparecer tanto no útero como nos ovários, trompas, bexiga e intestino, acompanhada de dor pélvica ou abdominal. Essa dor pode ser forte o suficiente para causar incapacidade interferindo negativamente nas suas atividades diárias. Aprender a conviver com essas dores pélvicas intensas é um grande desafio para as mulheres portadoras de endometriose, pois além delas esse quadro costuma cursar ainda com disfunções ginecológicas, alterações menstruais, alterações intestinais e urinárias cíclicas, o que limita aspectos da vida da paciente (CARAN J, et al., 2021).

Entender que apesar da doença não ser curável, ela também não é maligna e existe um tratamento. Essa doença é difícil para a maioria dessas pacientes e não são só os sintomas físicos que influenciam na vida destas; a dificuldade em relacionamentos, redução em atividades de lazer com comprometimento das atividades da vida cotidiana e interação social que antes eram prazerosas, além do desempenho profissional prejudicado, e absentismo devido a dor podem cursar com sintomas de ansiedade e depressão. Além disso, o pensamento catastrófico futuro, principalmente relacionado a possível infertilidade, é um fator que agrava ainda mais o estado mental dessas enfermas (RAMOS ELA, et al., 2018).

Este pensamento pode não ser compreendido por seus parceiros, afetando a relação conjugal e, associado à dispareunia, ocasiona diminuição da autoestima; Também influencia com prejuízo da vida sexual do casal e acarreta impacto negativo no parceiro. Sabemos que a sexualidade é um fenômeno que faz parte do bem-estar tanto físico quanto psicológico e social, por isso seu comprometimento acarreta repercussões que podem levar a disfunção sexual e ao fim do relacionamento (MARQUI ABT, et al., 2015).

A relação da endometriose com a infertilidade pode ser esclarecida a partir de mecanismos que juntos aumentam essa probabilidade de ocorrência, sendo a inflamação peritoneal a principal causa desses eventos patológicos, posto que o estado constante de estresse oxidativo prejudica a interação do ovócito com o espermatozoide; Componentes presentes no líquido peritoneal, como macrófagos, interleucinas 1 e 6 e TNFalfa, também são capazes de alterar a motilidade dos espermatozoides (DUARTE AN, 2021).

Alterações na anatomia pélvica explicaria a dificuldade de liberação do ovócito e sua captação pelas tubas uterinas uma vez que existem aderências que acabam prejudicando este evento, associado também à um desequilíbrio nas secreções de hormônios. A relação da endometriose com a infertilidade fica mais evidente quando a doença está avançada. Entretanto, a maioria das mulheres possui um grau leve ou moderado o que permite uma maior facilidade para engravidar (CALDEIRA TB, et al., 2017).

A associação da doença com a mulher moderna ocorre pelo fato das mulheres atuais priorizarem a sua formação profissional para depois pensarem em formar uma família. Assim, em consequência do adiamento da maternidade há redução das gestações e consequentemente maior número de ciclos menstruais. Com isso, observamos que mulheres com gestações tardias e grande intervalo entre a menarca e primeira gestação, têm risco aumentado para a endometriose que é uma das principais causas de infertilidade (GOMES JWF, et al., 2018).

Visando melhorar os cuidados com a saúde da mulher, o Ministério da Saúde (MS) desenvolveu o Protocolo de Atenção Básica para a Saúde da Mulher, incluindo o acolhimento às usuárias, a postura humanizada dos profissionais, e a escuta qualificada no que diz respeito a uma patologia ainda nova e que gera dúvidas à paciente. $O$ esclarecimento sobre a doença é fundamental no tratamento, assegurando confiança (ALVES TS, et al., 2021).

Apesar de ser uma inflamação crônica, até o momento, não existe uma teoria única que identifique e explique todos os aspectos clínicos dessa doença considerada multifatorial. As células endometrióticas têm capacidade de adesão fora da cavidade uterina, e essa sobrevivência é influenciada por fatores genéticos, disfunção do sistema imunológico, desregulação hormonal e capacidade de formação de novos vasos. Isso explica alguns possíveis mecanismos da sua etiofisiopatologia (STEFENON LP e BOSSOLANI GDP, 2020).

Apesar dessa regurgitação também ocorrer em mulheres sem a EDM, ela é mais comum em pacientes com a doença e esses implantes ocorrem pela influência de um ambiente hormonal favorável, pois 
diferentemente do tecido endometrial intrauterino, os implantes endometrióticos podem produzir aromatase, levando à produção de estrogênio extraovariano e de fatores imunológicos que não eliminariam essas células do local impróprio. A imunidade celular, especialmente a atividade das células exterminadoras naturais (natural killer cells) pode estar deficiente e a capacidade em reconhecer o tecido endometrial em locais anormais pode estar prejudicada. Isso permite que o tecido menstrual se implante e cresça no peritônio (ROMANO FB, et al., 2021; MELCHIOR HS, et al., 2019).

Outra teoria é a da metaplasia celômica, em que o mesotélio se transforma em tecido endometrial. Essa teoria advém das células endometriais que podem através de um processo de metaplasia, formar as lesões endometriais, ocorrendo por meio de um processo oncogênico. Ou seja, células totipotentes, com potencial de se transformar em qualquer tecido do corpo, forma um tecido histologicamente distinguível do endométrio normal. Isso explica os casos raros de mulheres que não menstruam e desenvolvem a doença (PERSOONS E, et al., 2020).

Para a medicina, a EDM é dividida em três grupos: endometriose peritoneal (considerada mais superficial), ovariana e profunda, que seria aquela com profundidade maior que $5 \mathrm{~mm}^{3}$, acometendo regiões mais profundas e de forma infiltrativa, como regiões reto-cervical, reto-sigmoide, ureteres e bexiga, sendo considerado a forma mais severa e de pior prognóstico (ROMANO FB, et al., 2021).

O diagnóstico precoce é a chave para o sucesso no tratamento da endometriose, porém, ainda é o maior desafio da doença. O espectro de apresentação da EDM é bastante variável, por isso ela pode se desenvolver de forma insidiosa e assintomática ou ainda apresentar manifestações de dor pélvica intensa ou sintomas característicos dos órgãos à distância afetados e produzir erro ou atraso no diagnóstico, em média de 6,7 anos (TORRES JISL, et al., 2021).

O atraso no diagnóstico pode ser devido a inúmeros fatores, o que mascara o quadro clínico, podendo ser confundido com outras patologias, como: doença inflamatória pélvica, miomatose uterina, adenomiose, tumores, além de afecções urológicas e gastrointestinais (CONCEIÇÃO HN, et al., 2019).

De acordo com a European Society of Human Reproduction and Embriology (ESHRE) o diagnóstico é evidenciado com base na história clínica, sempre correlacionando sinais e sintomas com o período menstrual. Essa patologia deve ser pensada para qualquer paciente em idade fértil que se queixa de dor menstrual progressiva ou de infertilidade (GEYTER CHD, et al., 2018).

O exame ginecológico pode apresentar-se normal, porém a presença de dor à movimentação do útero, crescimento do volume ovariano ou retroversão uterina é desconfiança da doença, mesmo não sendo específica. O espessamento do ligamento uterossacro, lesões violáceas na vagina ou as nodulações palpáveis no fórnice vaginal posterior são os indícios que sugerem endometriose profunda infiltrativa (CONCEIÇÃO HN, et al., 2019).

Já as técnicas de imagem, podem ser utilizadas a Ultrassonografia Transvaginal (USG-TV), Ressonância Magnética (RM) e Tomografia Computadorizada (TC). A USG-TV é o primeiro exame a ser solicitado, por ser mais acessível e barato, porém possui limitações quanto a visualização rigorosa da região da pelve e espaço subperitoneal, sendo muitas vezes ineficiente. A RM, devido ao alto custo, acaba não sendo tão solicitada, mas apresenta excelente visualização, em vários planos, para um diagnóstico mais preciso (CHALUB JP, et al., 2020).

Exames laboratoriais podem ser solicitados para excluir outras causas de dor pélvica, hemograma completo, dosagem de gonadotrofina coriônica humana, exame e culturas de urina, culturas vaginais e esfregaços do colo uterino podem ser realizados para excluir infecções ou complicações da gestação. Porém, o diagnóstico final padrão ouro é a laparoscopia, não é necessário a biópsia do tecido para confirmação, pois já há alta correlação entre os achados laparoscópicos e histológicos, sendo desnecessário a confirmação histológica. Então, esse procedimento permite ao médico uma visualização direta dos órgãos pélvicos, possibilita localização exata dos focos da doença e, quando indicado, remoção durante 0 ato cirúrgico (NOGUEIRA ACR, et al., 2018). 
O tratamento cirúrgico leva a erradicação dos focos da doença, e como o principal método de diagnóstico da endometriose é a laparoscopia, a realização diagnóstica-terapêutica é uma opção vantajosa. Porém, apesar de ser considerada progressiva, a endometriose pode permanecer estável e, em alguns casos, regredir sem tratamento (ROCHA AM, et al., 2018).

Os objetivos das terapias atuais são: reduzir a sintomatologia, promover a inativação dos implantes e aumentar a possibilidade de gestação, retardando a recorrência. O tratamento clínico, apesar de não ter eficácia para a cura, está indicado nas queixas álgicas, com bloqueio da menstruação por hormônios, em uso contínuo. Esse bloqueio hormonal pode ser feito por via oral, intramuscular, implantes ou dispositivo intrauterino. Contudo, esses métodos, apesar da ótima eficácia, não são a melhor opção para as pacientes que querem engravidar (CHALUB JP, et al., 2020).

Há prejuízo da qualidade de vida dessas mulheres, mesmo após o tratamento, por sentirem-se culpadas, sem a garantia de cura ou de que não haverá recidivas, o que resulta em manutenção de diversos transtornos já descritos, evidenciando a importância do diagnóstico precoce para o bom prognóstico (TORRES JISL, et al., 2021).

Além disso, como existe uma prevalência significativa de endometriose em casos de mulheres inférteis e essa associação aumenta com o estágio da doença, sua conduta deve ser feita de forma criteriosa. Ainda não existem evidências suficientes que afirmem o sucesso da concepção espontânea ou técnica nessas pacientes, contudo, a eficácia da Fertilização In Vitro (FIV), no contexto de pacientes com endometriose, está cada vez mais aceita nas últimas décadas. A Terapia de Reprodução Assistida é uma alternativa viável nesses casos, aumentando significativamente as chances de uma gravidez, tendo taxas de sucesso semelhante às pacientes não portadoras, pois, mesmo apresentando níveis avançados da doença, os ovócitos permanecem saudáveis nestas pacientes (ARAÚJO FWC e SCHMIDT DB, 2020).

A endometriose é uma doença de incerteza relacionada ao diagnóstico, em como a doença evolui e em relação ao seu futuro. Diante da complexidade dessa patologia, um acompanhamento psicoterápico para essas mulheres é de fundamental importância para o auxílio do tratamento e minimização dos problemas de saúde pública, uma vez que, essa realidade de diagnóstico e tratamento inserem essas pacientes em um contexto que predispõe o desenvolvimento de transtornos psicológicos. Sendo assim, a troca de experiências entre mulheres portadoras, e a cessação de dúvidas frequentes sobre a doença contribuem para melhoria da qualidade de vida (BENTO PADSS e MOREIRA MCN, 2018).

Além disso, a família e em especial o parceiro ajudam as mulheres durante o tratamento, contando com orientação e estímulo do profissional de saúde, levando-os a contribuir com uma participação ativa na superação dessa fase difícil que essas mulheres estão passando, principalmente no que diz respeito a tentativa de engravidar (ARAÚJO FWC e SCHMIDT DB, 2020).

A dor crônica intensa traz prejuízos em relacionamentos familiares, social e profissional o que leva muitas vezes ao isolamento, devido a pensamentos negativos ajuntados à diminuição da qualidade de vida. É fundamental o papel do profissional de saúde no pós-diagnóstico fortalecendo o empoderamento dessas mulheres no que diz respeito a informações necessárias para o entendimento dessa patologia e assim conseguir uma total adesão ao tratamento, sempre buscando melhorar seus aspectos biopsicossociais (RAMOS ELA, et al., 2018).

A endometriose é uma patologia considerada um problema de saúde pública devido ao descaso frente aos problemas enfrentados por essas pacientes, agregada a uma falta de investimento financeiro público acarretando um longo período até que se chegue a um diagnóstico definitivo, além da dificuldade de um tratamento adequado (TORRES JISL, et al., 2021).

A mudança no estilo de vida com a prática de atividade física e hábitos alimentares adotando uma dieta saudável, têm efeitos benéficos, e são uma alternativa terapêutica para melhorar a qualidade de vida dessas pacientes, uma vez que estes influenciam na patogênese da endometriose e consequentemente auxiliam no controle da sua manifestação clínica, sendo inseto ainda de efeitos colaterais, como o que é observado em alguns casos devido ao tratamento atual disponível (CHALUB JP, et al., 2020). 


\section{CONSIDERAÇÕES FINAIS}

A endometriose é uma patologia da sociedade moderna. A evolução da mulher, do lar para o mercado de trabalho, protelou a primeira gestação, assim como fortaleceu o seu papel social e a necessidade de manutenção da sua saúde mental. Mulheres com endometriose apresentam diminuição da qualidade de vida, tanto na saúde mental quanto no bem-estar emocional quando comparadas com a população geral, uma vez que a endometriose cursa com dismenorreia progressiva, algia pélvica crônica, infertilidade, dispareunia, alterações intestinais e urinárias cíclicas e seu tratamento envolve longa duração e associação com efeitos colaterais hormonais. Além disso, a endometriose atualmente é uma doença muito prevalente entre as mulheres, e que ainda representa um desafio para os ginecologistas por ser uma doença pouco conhecida na sua gênese e na maioria das vezes apresentar atraso no diagnóstico, sendo a falta de informação uma das principais queixas das portadoras desta patologia.

\section{REFERÊNCIAS}

1. ALVES TS, et al. Endometriose e sua relação com o câncer de ovário: uma revisão integrativa. Pesquisa, Sociedade e Desenvolvimento, 2021; 10(13): e14101320965.

2. ARAÚJO FWC, SCHMIDT DB. Endometriose um problema de saúde pública: revisão de literatura. Revista Saúde e Desenvolvimento, 2020; 14(18): 24-37.

3. BAETAS BV, et al. Endometriose e a qualidade de vida das mulheres acometidas. Revista Eletrônica Acervo Científico, 2021; 19: e5928.

4. BENTO PASS, MOREIRA MCN. Quando os olhos não veem o que as mulheres sentem: a dor nas narrativas de mulheres com endometriose. Physis: Revista de Saúde Coletiva, 2018; 28(3): e280309.

5. CHALUB JP, et al. Investigação sobre os aspectos nutricionais relacionados à endometriose. Research, Society and Development, 2020; 9(11): e65591110215.

6. CALDEIRA TB, et al. Infertilidade na endometriose: etiologia e terapêutica. HURevista, 2017; 43(2): 173-178, 2017.

7. CARAN J, et al. Escore embrionário graduado em embriões de mulheres inférteis com e sem endometriose peritoneal. Revista Brasileira de Ginecologia e Obstetrícia, 2021; 43(1): 28-34.

8. CONCEIÇÃO HN, et al. Endometriose: aspectos diagnósticos e terapêuticos. Revista Eletrônica Acervo Saúde, 2019; (24): e472.

9. DUARTE AN. Associação entre endometriose e infertilidade feminina: uma revisão de literatura. Acta Elit Salutis, 2021; 4(1): $1-12$.

10. GEYTER C, et al. Resultados das técnicas de reprodução assistida em 2014 obtidos dos registros da Sociedade Europeia Humana e Embriologia (ESHRE). Reprodução humana, 2018; 33(9): 1586-1601.

11. GOMES JWF, et al. Ressecção de endometriose ureteral com reimplante uretrovesical por videolaparoscopia. Revista Urominas, 2018; 5(12): 36-37.

12. MARQUI ABT, et al. Disfunção sexual em endometriose: uma revisão sistemática. Medicina (Ribeirão Preto), 2015; 48(5): $478-490$.

13. MELCHIOR HS, et al. Endometriose: aspectos gerais e associação a infertilidade. Revista Terra \& Cultura: Cadernos de Ensino e Pesquisa, 2019; 34(67): 95-106.

14. MORAIS HB, et al. Impactos negativos da endometriose na qualidade de vida da mulher acometida: uma revisão integrativa de literatura. Brazilian Medical Students, 2021; 5(8): 1-10.

15. MORAIS RL, ROSAL MA. Preditores de endometriose em mulheres atendidas em um Hospital Universitário. Jornal de Ciências da Saúde do Hospital Universitário da Universidade Federal do Piauí, 2021; 4(1): 24-39.

16. NOGUEIRA ACR, et al. Tratamento da endometriose pélvica: uma revisão sistemática. Revista Científica UNIFAGOC-Saúde, 2018; 3(2): 38-43.

17. OLIVEIRA LAF, et al. Relação entre ocorrência de endometriose e sofrimento psíquico. Revista Brasileira em Promoção da Saúde. Fortaleza, 2018; 31(4): 1-6.

18. PERSOONS E, et al. Mimicking Sampson's Retrograde Menstrual Theory in Rats: A New Rat Model for Ongoing Endometriosis-Associated Pain. International journal of molecular sciences, 2020; $21(7): 2326$.

19. RAMOS ELA, et al. Mulheres convivendo com endometriose: percepções sobre a doença. Ciência \& Saúde, 2018; 11(3): 190-197.

20. ROCHA AM, et al. Impacto tardio do tratamento laparoscópico da Endometriose Profunda Infiltrativa com ressecção segmentar colorretal. ABCD. Arquivos Brasileiros de Cirurgia Digestiva (São Paulo), 2018; 31(4): e1406.

21. ROMANO FB, et al. Recidiva de endometriose após histerectomia: revisão integrativa. Revista Eletrônica Acervo Saúde, $2021 ; 13(8):$ e 8545.

22. SILVA CM, et al. Experiências das mulheres quanto às suas trajetórias até o diagnóstico de endometriose. Escola Anna Nery, 2021; 25(4): e20200374.

23. SOUZA GKT, et al. Endometriose $x$ infertilidade: revisão de literatura. Encontro de Extensão, Docência e Iniciação Científica (EEDIC), 2017; 3(1): 1-4.

24. SOUZA TSB, et al. Papel da Enfermagem frente a portadoras de Endometriose e depressão. Revista de enfermagem UFPE online, 2019; 13(3): 811-818.

25. STEFENON LP, BOSSOLANI GDP. Os desafios no diagnóstico e tratamento da endometriose. Revista Saúde Viva Multidisciplinar da AJES, 2020; 3(4): 1.

26. TOMÁS C, METELLO JL. Endometriose e infertilidade-onde estamos? Acta Obstétrica e Ginecológica Portuguesa. 2019; 13(4): 235-241.

27. TORRES JISL, et al. Endometriose, dificuldades no diagnóstico precoce e a infertilidade feminina: Uma Revisão. Research, Society and Development, 2021; 10(6): e6010615661.

28. YELA DA, et al. Qualidade de vida de mulheres com endometriose profunda: Estudo de corte transversal. Revista Brasileira de Ginecologia e Obstetrícia, 2020; 42(2): 90-95. 